PREPARED FOR THE U.S. DEPARTMENT OF ENERGY, UNDER CONTRACT DE-AC02-76CH03073

PPPL-3549

PPPL-3549

UC-70

Parametric Excitations of Fast Plasma Waves

by Counter-propagating Laser Beams

by

G. Shvets and N.J. Fisch

March 2001

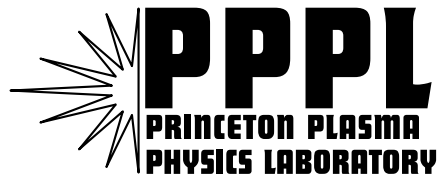

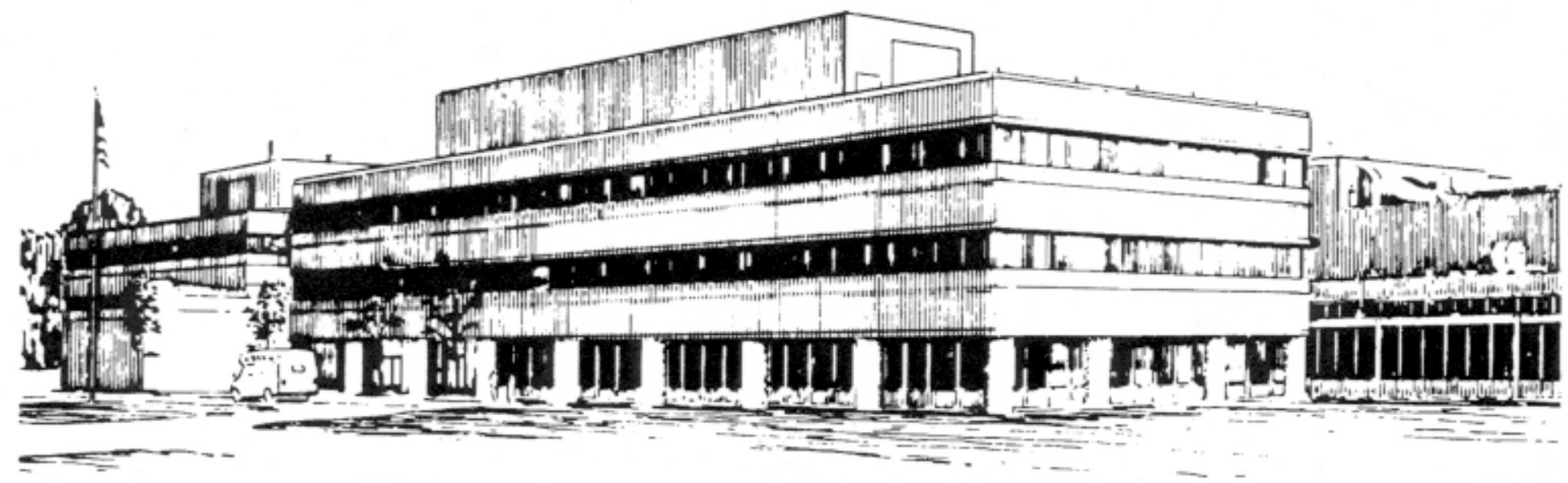

PRINCETON PLASMA PHYSICS LABORATORY PRINCETON UNIVERSITY, PRINCETON, NEW JERSEY 


\section{PPPL Reports Disclaimer}

This report was prepared as an account of work sponsored by an agency of the United States Government. Neither the United States Government nor any agency thereof, nor any of their employees, makes any warranty, express or implied, or assumes any legal liability or responsibility for the accuracy, completeness, or usefulness of any information, apparatus, product, or process disclosed, or represents that its use would not infringe privately owned rights. Reference herein to any specific commercial product, process, or service by trade name, trademark, manufacturer, or otherwise, does not necessarily constitute or imply its endorsement, recommendation, or favoring by the United States Government or any agency thereof. The views and opinions of authors expressed herein do not necessarily state or reflect those of the United States Government or any agency thereof.

\section{Availability}

This report is posted on the U.S. Department of Energy's Princeton Plasma Physics Laboratory Publications and Reports web site in Calendar Year 2001. The home page for PPPL Reports and Publications is: http://www.pppl.gov/pub_report/

DOE and DOE Contractors can obtain copies of this report from:

U.S. Department of Energy

Office of Scientific and Technical Information

DOE Technical Information Services (DTIS)

P.O. Box 62

Oak Ridge, TN 37831

Telephone: (865) 576-8401

Fax: (865) 576-5728

Email: reports@adonis.osti.gov

This report is available to the general public from:

National Technical Information Service

U.S. Department of Commerce

5285 Port Royal Road

Springfield, VA 22161

Telephone: 1-800-553-6847 or

(703) 605-6000

Fax: (703) 321-8547

Internet: http://www.ntis.gov/ordering.htm 


\title{
Parametric excitations of fast plasma waves by counter-propagating laser beams
}

\author{
G. Shvets and N. J. Fisch \\ Princeton Plasma Physics Laboratory, Princeton University, Princeton, NJ 08543
}

\begin{abstract}
Short and long wavelength plasma waves can become strongly coupled in the presence of two counter-propagating laser pump pulses detuned by twice the cold plasma frequency $\omega_{p}$. What makes this four-wave interaction important is that the growth rate of the plasma waves occurs much faster than in the more obvious co-propagating geometry.
\end{abstract}

An important nonlinear process in plasma physics is the beatwave excitation of the electron plasma wave using high-frequency lasers, with applications including plasma heating and current drive [1,2], studying and controlling the ionosphere [3], and accelerating charged particles $[4,5]$. In this Letter we demonstrate that a fast plasma wave with phase velocity close to the speed of light can be generated by crossing two counter-propagating laser beams, which are detuned by $|\Delta \omega| \approx 2 \omega_{p}$, where $\omega_{p}=\left(4 \pi e^{2} n_{0} / m\right)^{1 / 2}$ is the plasma frequency, $-e$ and $m$ are the electron charge and mass, and $n_{0}$ is the plasma density. The counter-propagating geometry departs from the geometry employed in the traditional plasma beatwave accelerator (PBWA) approach to generating fast plasma waves for particle acceleration, which utilizes co-propagating laser pulses [6] detuned by $\Delta \omega=\omega_{p}$.

That a plasma wave can be driven unstable by the $2 \omega_{p}$ beatwave was originally proposed by Rosenbluth and Liu (RL) [7], who calculate a growth rate of a fast plasma wave $\gamma_{R L} \approx$ $\omega_{p} a_{0} a_{1} / 2$ (co-propagating lasers). Note that this decay is high-order, with growth rate going as pump amplitude squared. Thus, for pump waves of sub-relativistic intensity, i.e. $a_{0}, a_{1} \ll 1$, this decay instability is too slow to be of great practical interest. 
What we propose here is that a counter-propagating pump geometry results in a growth rate also second order in the pump amplitude, but strongly enhanced by the factor $2 \omega_{0}^{2} / \omega_{p}^{2}$. We consider the four-wave interaction, in which the four participating waves are: the counterpropagating lasers, and two plasma waves, one (slow) with about twice the laser wavenumber, and one (fast) with the small wavenumber $\left(\omega_{p} / c\right)$. For the similar reason why Raman backscattering is much faster than Raman forward scattering, here the counter-propagating geometry enhances the growth rate, but now in the much different context of a four-wave interaction in which there is decay to a fast plasma wave capable of particle acceleration.

To proceed, consider then the interaction of two counter-propagating laser beams (labeled by 0 and 1 ), with the corresponding normalized vector potentials given by $\vec{a}_{0,1}=$ $a_{0,1}\left(\vec{e}_{ \pm} \exp \left(i \theta_{0,1}\right)+c . c.\right)$, where $\vec{e}_{+(-)}=\left(\vec{e}_{x} \pm i \vec{e}_{y}\right) / 2, \theta_{0}=k_{0} z-\omega_{0} t$, and $\theta_{1}=k_{1} z+\omega_{1} t$. We assume that the duration of the forward-moving laser pulse is short (several plasma periods) and the duration of the backward-moving pulse is twice the length of the plasma. Tenuous plasma $\omega_{p} \ll \omega_{0}$ is assumed, ensuring that the lasers propagate almost as in vacuum: $v_{g 0} \approx c$ and $\left|\vec{k}_{0}-\vec{k}_{1}\right| \approx 2 k_{0}$. The 4-wave instability we consider involves a short-wavelength (slow) and a long-wavelength (fast) plasma wave. The wavenumber of the slow wave is $k_{s}=2 k_{0}-k_{p}$. The wavenumber of the fast wave $k_{p}$ is determined by the group velocity of the short pulse $v_{g}: k_{p}=\omega_{p} / v_{g} \approx \omega_{p} / c$.

Just as for the co-propagating geometry, the time-averaged ponderomotive force $\vec{F}=$ $-m c^{2} \nabla\left(\vec{a}_{0} \cdot \vec{a}_{1}\right) \approx 2 k_{0} m c^{2} \sin \left(2 k_{0} z-\Delta \omega\right)$ due to the pump lasers drives the plasma waves:

$$
\ddot{\xi}+\omega_{p}^{2} \xi=i k_{0} c^{2} a_{0} a_{1} e^{i\left[\Delta \omega t-2 k_{0} z\right]}+c . c .,
$$

where $\xi \equiv z-z_{0}$ is the Lagrangian electron displacement. For the co-propagating geometry, RL [7] used a single-wave ansatz for the plasma electron displacement $\xi=$ $A(t) \sin \left[k z-\omega_{p} t+\phi(t)\right]$. The single-wave ansatz used by RL, however, is not sufficiently general for the case of counter-propagating lasers. Consider instead then the two-wave ansatz:

$$
\xi=A_{f} \sin \left[k_{p} z_{0}-\omega_{p} t+\phi_{f}\right]+A_{s} \sin \left[k_{s} z_{0}-\omega_{p} t+\phi_{s}\right]
$$


where $A_{f}\left(\phi_{f}\right)$ and $A_{s}\left(\phi_{s}\right)$ are the amplitudes (phases) of the fast and slow plasma waves. For simplicity, in the analytic calculation we assume monochromatic laser waves. Shortpulse effects are numerically treated later in the paper. Substituting $z=z_{0}+\xi$, where $\xi$ is given by Eq. (2), into the RHS of Eq. (1) yields

$$
\begin{aligned}
& \frac{\partial^{2} \xi}{\partial t^{2}}+\omega_{p}^{2} \xi=i k_{0} c^{2} a_{0} a_{1} \sum_{k, l}(-1)^{k+l} J_{k}\left(2 k_{0} A_{f}\right) J_{l}\left(2 k_{0} A_{s}\right) \\
& e^{i k\left[k_{p} z_{0}-\omega_{p} t+\phi_{f}\right]} e^{i l\left[\left(k_{s} z_{0}-\omega_{p} t+\phi_{s}\right]\right.} e^{i\left[\Delta \omega t-2 k_{0} z_{0}\right]}+c . c .
\end{aligned}
$$

where $J_{k, l}$ are Bessel functions, and $\Delta \omega=\omega_{0}-\omega_{1} \equiv 2 \omega_{p}+\delta \omega$. In writing the RHS of Eq. (3), we used the identity $e^{i \alpha \sin \phi}=\sum_{k} J_{k}(\alpha) e^{i k \phi}$. A set of purely time-dependent equations can now be obtained by separating the $z_{0}$ dependent terms on both sides of Eq. (3). Thus, substituting Eq. (2) into LHS of Eq. (3) and matching the corresponding harmonics of $k_{p} z_{0}$ and $k_{s} z_{0}$ on both sides of the equation, we can write for the $(k=0, l=1)$ and $(k=1, l=0)$ terms the following:

$$
\begin{aligned}
& \frac{\partial \phi}{\partial t}=\delta \omega-\frac{\Omega_{B}^{2}}{4} \omega_{p} G\left(A_{f}, A_{s}\right) \sin \phi \\
& \frac{\partial\left(k_{0} A_{f}\right)}{\partial\left(\omega_{p} t\right)}=\frac{\Omega_{B}^{2}}{4} J_{0}\left(2 k_{0} A_{f}\right) J_{1}\left(2 k_{0} A_{s}\right) \cos \phi \\
& \frac{\partial\left(k_{0} A_{s}\right)}{\partial\left(\omega_{p} t\right)}=\frac{\Omega_{B}^{2}}{4} J_{1}\left(2 k_{0} A_{f}\right) J_{0}\left(2 k_{0} A_{s}\right) \cos \phi,
\end{aligned}
$$

where $\phi=\phi_{s}+\phi_{f}+\pi / 2+\delta \omega t, \Omega_{B}^{2}=4 a_{0} a_{1} \omega_{0}^{2} / \omega_{p}^{2}$ is the square of the electron bounce frequency in the optical lattice created by the interference of the counter-propagating lasers, and

$$
G\left(A_{f}, A_{s}\right)=\frac{J_{0}\left(2 k_{0} A_{f}\right) J_{1}\left(2 k_{0} A_{s}\right)}{k_{0} A_{f}}+\frac{J_{1}\left(2 k_{0} A_{f}\right) J_{0}\left(2 k_{0} A_{s}\right)}{k_{0} A_{s}}
$$

Higher order Bessel terms are of the same order in the plasma wave amplitudes $2 k_{0} A_{f, s}$, and are assumed small.

At resonance $(\delta \omega=0)$, the relative phase $\phi$ locks at $\phi=0$. For small amplitudes of the plasma waves, $2 k_{0} A_{f, s} \ll 1$, Eqs. $(5,6)$ can then be linearized, predicting the simultaneous exponential amplification of the fast and slow waves with the growth rate $\Omega_{i}=\omega_{0}^{2} a_{1} a_{0} / \omega_{p}$. In 
a nutshell, this is the principal result of this work: fast plasma wave capable of accelerating relativistic particles can be produced with a high temporal growth rate $\Omega_{i}$. This growth rate is much higher than that predicted by RL for the co-propagating lasers: $\Omega_{i} / \gamma_{R L} \approx 2 \omega_{0}^{2} / \omega_{p}^{2}$. The fast wave $A_{f}$ grows so rapidly because it is parametrically coupled to the slow wave $A_{s}$. The coupling mechanism is the ponderomotive force due to the counter-propagating optical mixing of the laser beams.

An important practical issue is the sensitivity of the instability to the deviation from the exact two-plasmon resonance $\delta \omega$. For the finite frequency detuning from resonance $\delta \omega \neq 0$, there is an intensity threshold: phase-locking takes place only if $\Omega_{B}^{2} / 2>\delta \omega / \omega_{p}$. Here, again, the counter-propagating geometry offers an advantage over the co-propagating case: the intensity threshold is given by

$$
\sqrt{I_{0} I_{1}}\left[\mathrm{~W} / \mathrm{cm}^{2}\right]=1.4 \times 10^{-3}\left(\delta \omega / \omega_{p}\right) n_{0}\left[\mathrm{~cm}^{-3}\right]
$$

For example, if the laser wavelengths are $\lambda_{0}=0.8 \mu \mathrm{m}$ and $\lambda_{1}=1.0 \mu \mathrm{m}$, and plasma density is $n_{0}=10^{19} \mathrm{~cm}^{-3}$ (corresponding to $\omega_{0}-\omega_{p}=2.5 \omega_{p}$ ), the geometric mean of the laser intensities should exceed the threshold value of $8.0 \times 10^{15} \mathrm{~W} / \mathrm{cm}^{2}$. Since this threshold is not too high, the instability is quite robust to the plasma inhomogeneity and detuning errors.

Equations $(4,5,6)$ can be simplified by noting that, from the last two equations, $J_{0}\left(2 k_{0} A_{f}\right) / J_{0}\left(2 k_{0} A_{s}\right)=$ const. If both waves start out negligibly small, the constant is equal to unity, and one can assume that $A_{f}=A_{s}$ at all times. This assumption is only meaningful when the instability significantly amplifies both $A_{s}$ and $A_{f}$, so that the small absolute difference of the initial amplitudes is unimportant. The equations for the phase and the normalized amplitude $u=2 k_{0} A_{s}=2 k_{0} A_{f}$ become

$$
\begin{aligned}
& \dot{\phi}=\left(\delta \omega / \omega_{p}\right)-\Omega_{B}^{2} \frac{J_{0}(u) J_{1}(u)}{u} \sin \phi \\
& \dot{u}=\frac{\Omega_{B}^{2}}{2} J_{0}(u) J_{1}(u) \cos \phi,
\end{aligned}
$$

where the dot indicates a derivative with respect to $\omega_{p} t$. The conserved invariant of Eqs. $(8,9)$ is $\mathcal{H}=\Omega_{B}^{2} u^{2} \sin \phi-2\left(\delta \omega / \omega_{p}\right) F(u)$, where 


$$
F(u)=\int_{0}^{u} d x \frac{x^{2}}{J_{0}(x) J_{1}(x)} .
$$

Note is that $F(u)$ diverges for $u \rightarrow \mu_{0}$, where $\mu_{0}=2.405$ is the first zero of $J_{0}$.

For the excitation which starts out infinitesimally small $\mathcal{H} \approx 0$, and the $\sin \phi$ can be expressed in terms of the amplitude $u$. The expression for the $\cos \phi$ is then substituted into Eq. (9):

$$
\dot{u}=\frac{J_{0}(u) J_{1}(u)}{2}\left[\Omega_{B}^{4}-\frac{4 F^{2}(u)(\delta \omega)^{2}}{u^{4} \omega_{p}^{2}}\right]^{1 / 2} .
$$

Equation (10) gives the trajectory of the wave amplitude as a function of time. The plus (minus) sign corresponds to the increasing (decaying) portions of the trajectory. For a finite detuning $\delta \omega$, the "motion" of $u$ is periodic between its initial starting value $u_{0}$ and the maximum value $u_{\max }$.

For a perfect laser detuning $\delta \omega=0$, the mode amplitude has a stable attractor at $u=\mu_{0}$. Since $\mu_{0}>1, \mathrm{Eq}$. (3) no longer holds because of the breaking of the slow wave [8]. For $0<\left(\delta \omega / \omega_{p}\right)<\Omega_{B}^{2} / 2$ the amplitude $u$ oscillates periodically between its initially small value $u_{0}$ and $u_{\max }<\mu_{0}$ which is found by solving the equation $F\left(u_{\max }\right) / u_{\max }=\Omega_{B}^{2} \omega_{p} / 2 \delta \omega$. This equation has no solutions for $\left(\delta \omega / \omega_{p}\right)<\Omega_{B}^{2} / 2$, i. e. there is no instability. Defining $\delta \omega$ according to $\Omega_{B}^{2} / 2=(1+\epsilon)\left(\delta \omega / \omega_{p}\right)$, we plotted in Fig. 1 the temporal evolution of $u$ for a fixed $\Omega_{B}=1$ and three different detunings corresponding to $\epsilon=0.2,0.1,0.05$.

Analytic progress can be made in the limit of $u<1$, which is, in any case, the applicability limit of Eq. (3). Then $F(u)=u^{2}+3 / 16 u^{4}+\ldots$, and the maximum amplitude can be evaluated as $u_{\max }=4 \sqrt{\epsilon / 3}$. The oscillation period is given by $(\delta \omega) T=8 \sqrt{2 / 3} \ln \left[2 u_{\max } / u_{0}\right] / u_{\max }$, where $u_{0} \ll u_{\max }$ is the initial mode amplitude. Figure 1 confirms that the smaller is the peak amplitude of the wave, the longer is the oscillation period.

The physics of the amplitude oscillation can be understood as follows. Initially, $u$ is very small, and since the ratio $F(u) / u^{2}$ is approximately a constant, the relative phase is locked at a constant $\phi=\sin ^{-1} 2\left(\delta \omega / \omega_{p}\right) / \Omega_{B}^{2}$. As $u$ undergoes an exponential growth, the phase "unlocks" and drifts towards $\phi=\pi / 2$, at which time the amplitude peaks at $u=u_{\max }$ and 
starts dropping. After the amplitude drops to its initial value $u_{0}$, the phase locks again, and the process repeats.

While the fastest instability corresponds to $\Delta \omega=\omega_{s}+\omega_{f}$, it is instructive to understand qualitatively how two plasma oscillations $\omega_{s}$ and $\omega_{f}$ (fast and slow) can become strongly coupled by a beatwave which has a frequency $\Delta \omega \neq \omega_{s}+\omega_{f}$. It turns out that both $\omega_{f}$ and $\omega_{s}$ are shifted from $\omega_{p}$ in the presence of the lasers. The simplified description of the instability, expressed by Eqs. (4-6), predicts that the frequency shifts are proportional to $\left(a_{0} a_{1}\right)^{2}$. Indeed, consider the small-intensity regime $\left(\delta \omega / \omega_{p}\right) \gg \Omega_{B}^{2} / 2$. Then using $\phi \approx(\delta \omega) t$ and expanding Bessel functions to the lowest order in $A_{s, f}$, it can be shown from that both $\phi_{s}$ and $\phi_{f}$ acquire a time-averaged drift $\dot{\phi}_{s, f}=-\delta \Omega_{s, f}$, where $\delta \Omega_{s}=\delta \Omega_{f}=\Omega_{B}^{4} /\left(32 \delta \omega / \omega_{p}\right)$. Therefore, in the presence of the nonresonant beatwave the frequencies of both modes are shifted in the direction of $(\delta \omega)$. A rough estimate of the instability threshold can be obtained by requiring that $\delta \Omega_{s}+\delta \Omega_{f}=\left(\delta \omega / \omega_{p}\right)$. This results in $\Omega_{B}^{2}=4\left(\delta \omega / \omega_{p}\right)$, overestimating the earlier obtained expression for the intensity threshold by a factor 2. As shown below, there is an additional mechanism of shifting the frequency of the slow plasma wave via backscattering the short laser pulse. This frequency shifting can significantly modify the threshold intensity.

Since multiple plasma and laser waves are involved, Eqs. (4-6) describe the instability only approximately. Some of the missed effects are: (i) plasma perturbation driven at frequency $\Delta \omega$; (ii) modification of $a_{1}$ by the backscattering of $a_{0}$ off this driven density perturbation; (iii) the renormalization of the slow wave frequency due to its interaction with the short laser pulse. Therefore, we supplement the above calculation by a more rigorous two-scale particle simulation, which takes advantage of the scale separation between the short period of the slow plasma wave and a much longer period of the fast wave. We also assume for simplicity that the forward propagating laser pulse $a_{0}$ is much shorter than $a_{1}$.

The small-scale dynamics of the plasma electrons is characterized by their location (or phase) $\theta_{j}=\theta_{0}+\theta_{1} \approx 2 k_{0} z_{j}$ inside the optical lattice produced by the interference of the two lasers. Equations of motion for the $j^{\prime}$ s electron in a reference frame moving with the short pulse are described in Refs. [9,10]: 


$$
\ddot{\theta}_{j}+\Omega_{B}^{2} \sin \left(\theta_{j}-\Delta_{0} \zeta\right)=-\sum_{l=1}^{\infty} \hat{n}_{l} e^{i l \theta_{j}}-\tilde{e}_{z}+\text { c. c. },
$$

where a dot denotes a derivative with respect to $\zeta=\omega_{p}(t-z / c), \hat{n}_{l}=i\left\langle e^{-i l \theta_{j}} / l\right\rangle_{\lambda_{0} / 2}$ is the l-th harmonic of the small-scale electron plasma wave averaged over one lattice period, and $\Delta_{0}=\Delta \omega_{0} / \omega_{p}$. The global electric field $\tilde{e}_{z}=2 \omega_{0} e E_{z} / m c \omega_{p}^{2}$ is generated owing to the average momentum deposition from the lasers into the plasma [11]. In normalized units, equations for $\tilde{e}_{z}$ and $a_{1}$ can be written as

$$
\frac{\partial \tilde{e}_{z}}{\partial \zeta}=\left\langle\dot{\theta}_{j}\right\rangle_{\lambda_{0} / 2}, \quad \frac{\partial a_{1}}{\partial \zeta}=-i \frac{\omega_{p} a_{0}^{*}}{4 \omega_{0}}\left\langle e^{-i \theta_{j}}\right\rangle_{\lambda_{0} / 2}
$$

Equations $(11,12)$, supplemented by the initial conditions at $\zeta=-\infty$, are numerically solved using macro-particles. As an initial condition, we assume that at $\zeta=-\infty$ plasma is uniform ( $\hat{n}_{l}=0$ for all $l$ ) and stationary $\left(\dot{\theta}_{j}=0\right.$ for all $j$ ), and that a small initial fast plasma wave is present $\left(\tilde{e}_{z}=\tilde{e}_{0}\right)$. The presence of a much larger plasma wave inside the short pulse (taken here in the form $\left.a_{0}=0.5 \bar{a}_{0}\left[\tanh \left(-\zeta / \tau_{L}\right)+1\right]\right)$ indicates an instability.

The fast electric field $E_{z}$ obtained by integrating Eqs. $(11,12)$ is shown in Fig. 2 for two sets of laser field amplitudes $a_{0}$ and $a_{1}$. Simulation parameters are $\omega_{0} / \omega_{p}=10, \omega_{0}-\omega_{1}=$ 2.5 $\omega_{p}$, and $\tilde{e}_{0}=10^{-3}$. In Fig. 2(a) $a_{0}=a_{1}=0.06$ were assumed fixed. Evolving $a_{1}$ according to the second Eq. (12) did not result in any significant change of $E_{z}$. We also simulated the case of the fixed $a_{0}=0.19$ and $a_{1}=0.015$, which did not show any instability since in this case $\Omega_{B}^{2}$ is smaller then in Fig. 2(a). However, when $a_{1}$ was self-consistently evolved, a large electric field was excited, as shown in Fig. 2(b). This result is a manifestation of the physics which was not included in the above two-wave analysis which predicted that the threshold for the instability is determined by the frequency detuning $\delta \omega$ and $\Omega_{B}^{2}=4 a_{0} a_{1} \omega_{0}^{2} / \omega_{p}^{2}$, which only depends on the product of the laser amplitudes, not on the individual amplitudes.

As was explained earlier, the instability threshold arises because the finite $\Omega_{B}^{2}$ is needed to shift the frequencies of the fast and slow plasma waves to compensate for the frequency detuning $\delta \omega$. However, there may be other mechanisms of frequency shifting unaccounted for by the two-wave treatment. In particular, it follows from Eq. (12) that a slow wave with 
amplitude $\hat{n}_{1} \sim e^{-i \zeta}$ excites a backward wave $\delta a_{1}=\omega_{p} a_{0}^{*} \hat{n} / 4 \omega_{0}\left(\Delta_{0}-1\right)$, which then forms a beatwave with $a_{0}$ and acts back on the plasma electrons. Substituting $\delta a_{1}$ into Eq. (11), obtain an additional frequency shift of the slow plasma wave $\delta \Omega_{s}^{+}=\omega_{p}^{2}\left|a_{0}\right|^{2} / 4 \omega_{0}\left(\Delta \omega-\omega_{p}\right)$. For the simulation parameters of Fig. 2(b), this additional frequency shift, independent of $a_{1}$, effectively reduces the $\delta \omega=0.5 \omega_{p}$ frequency mismatch. Hence, $\Omega_{B}^{2}$ required to bridge the remaining gap is reduced as well. For the simulation parameters of Fig. 2(a) this reduction was negligible because of the smallness of $a_{0}^{2}$.

The relatively modest intensity threshold, given by Eq. (7), can be further lowered by employing a chirped laser pulse. Frequency chirp $\delta \omega(\zeta)$ also provides the benefit of suppressing the Raman backscattering of the more intense short pulse which can evolve from noise [12]. In Fig. 3 we plotted the amplitudes of the fast and slow plasma waves, $\tilde{e}_{z}$ and $\left\langle\cos \theta_{j}\right\rangle$, for a linearly-chirped Gaussian pulse. Assuming that $\lambda_{1}=1 \mu \mathrm{m}$, the central frequency of the laser $\omega_{0}=\omega_{1}+2.35 \omega_{p}$ corresponds to $\lambda_{0}=810 \mathrm{~nm}$, and the plasma frequency $\omega_{p} / \omega_{1}=0.1$ corresponds to $n_{0}=10^{19} \mathrm{~cm}^{-3}$, the pulse profile is as follows: $a_{0}=0.15 \exp \left[-\zeta^{2} / 2 \tau_{L}^{2}\right]$ with $\tau_{L}=25(160 \mathrm{fs} \mathrm{FWHM})$ and $d \delta \omega / d \zeta=-9.5 \times 10^{-3} \omega_{p}(3 \%$ bandwidth $)$. The initial fast plasma wave $\tilde{e}_{0}=10^{-3}$ and $a_{1}=0.0165$ have been assumed. In this example an accelerating plasma field of up to $9 \mathrm{GeV} / \mathrm{m}$ is generated.

In conclusion, we showed that large-amplitude fast plasma waves might be very effectively excited by two counter-propagating laser pulses detuned by approximately two plasma frequencies. In this arrangement, a slow plasma wave is incidentally excited, which is very effective in coupling the laser energy to the very useful for particle acceleration fast plasma wave.

This work was supported by the DOE Division of High Energy Physics. 


\section{REFERENCES}

[1] V. Stefan, B. I. Cohen, and C. Joshi, Science 243, 494 (1989).

[2] J. T. Mendonca and R. M. O. Galvao, J. PLasma Physics 35, 483 (1986); B. I. Cohen et. al., Nucl. Fusion 28, 1519 (1988).

[3] A. Y. Wong et. al., J. Geophys. Res. 86, 7718 (1981).

[4] T. Tajima and J. M. Dawson, Phys. Rev. Lett. 43, 267 (1979).

[5] M. J. Everett et. al., Phys. Rev. Lett. 74, 2236 (1995).

[6] C. Clayton et. al., Phys. Rev. Lett. 70, 37 (1993); F. Amiranoff et. al., Phys. Rev. Lett. 74, 5220 (1995).

[7] M. N. Rosenbluth, C. S. Liu, Phys. Rev. Lett. 29, 701 (1972).

[8] J. Dawson, Phys. Rev. 113, 383 (1959).

[9] G. Shvets, J. S. Wurtele, and B. A. Shadwick, Phys. Plasmas, 4, 1872 (1997).

[10] G. Shvets, N. J. Fisch, A. Pukhov, and J. Meyer-ter-Vehn, Phys. Rev. Lett. 81, 4879 (1998).

[11] G. Shvets, N. J. Fisch, A. Pukhov, and J. Meyer-ter-Vehn, Phys. Rev. E 60, 2218 (1999).

[12] V. M. Malkin, G. Shvets and N. J. Fisch, Phys. Rev. Lett. 84, 1208 (2000). 


\section{FIGURES}

FIG. 1. Fast and slow wave amplitude $u=2 k_{0} A_{f}$ as functions of time for three detunings: $\Omega_{B}^{2} / 2=(1+\epsilon)\left(\delta \omega / \omega_{p}\right)$. Initial excitation $u_{0}=10^{-3}, \Omega_{B}^{2}=1$

FIG. 2. Solid line: fast electric field $\tilde{e}_{z}$, dashed line: normalized intensity of short pulse $a_{0 n}^{2}$. (a) $a_{0}=a_{1}=0.06$, fixed $a_{1}$; (b) $a_{0}=0.19, a_{1}(\zeta=0)=0.015$, and $a_{1}$ is solved for from Eq. (12)

FIG. 3. Solid line: fast electric field $\tilde{e}_{z}$, long-dashed line: normalized intensity of short pulse $a_{0 n}^{2}$, dashed line: density bunching of the slow plasma wave $\operatorname{Re}\left(\hat{n}_{1}\right)=\left\langle\cos \theta_{j}\right\rangle$. Rapidly-varying part part of $\hat{n}_{1}$ is the driven plasma response inside the laser pulse. 


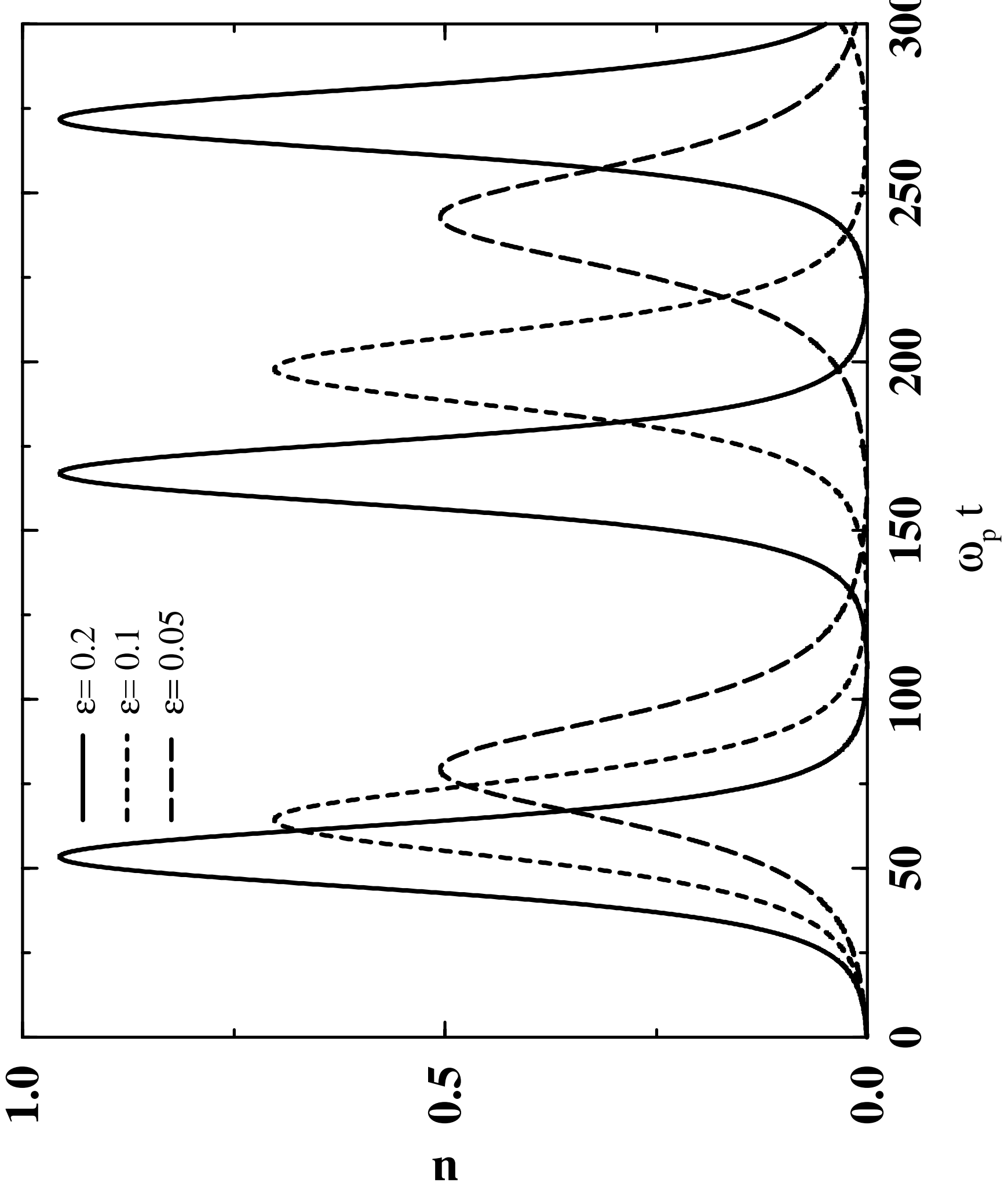




\section{Normalized Intensity}

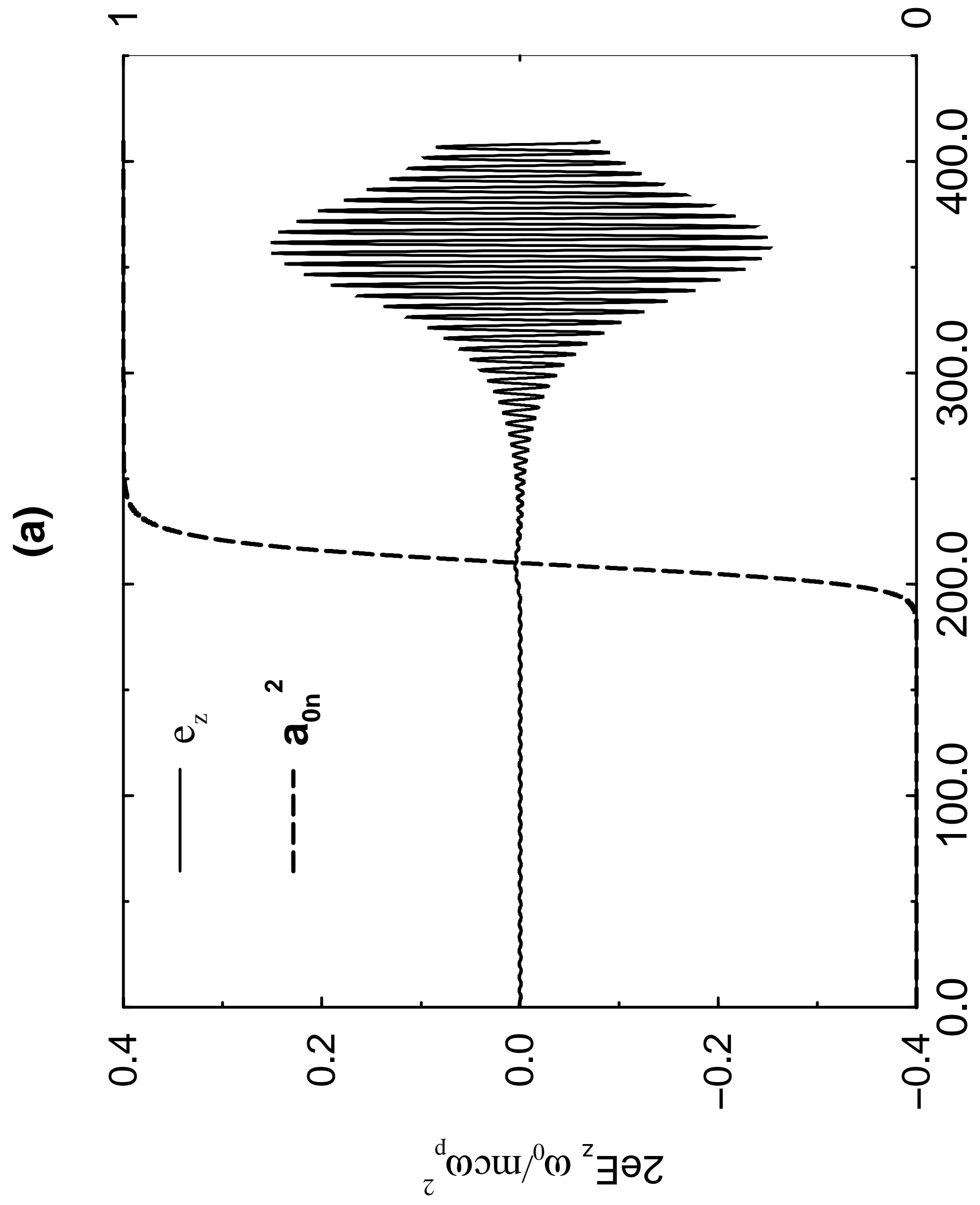




\section{Normalized Intensity}

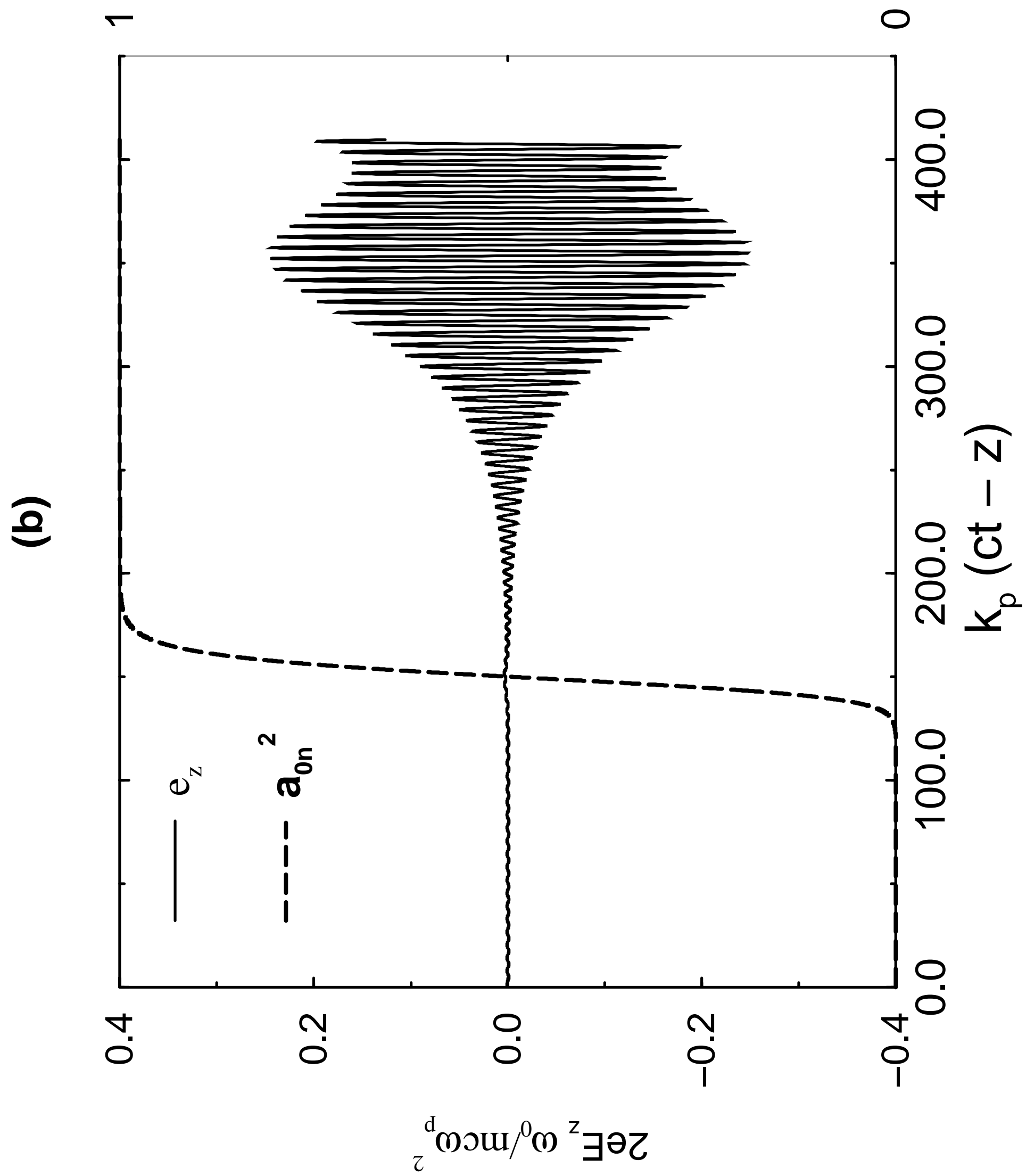




\section{Normalized Intensity}

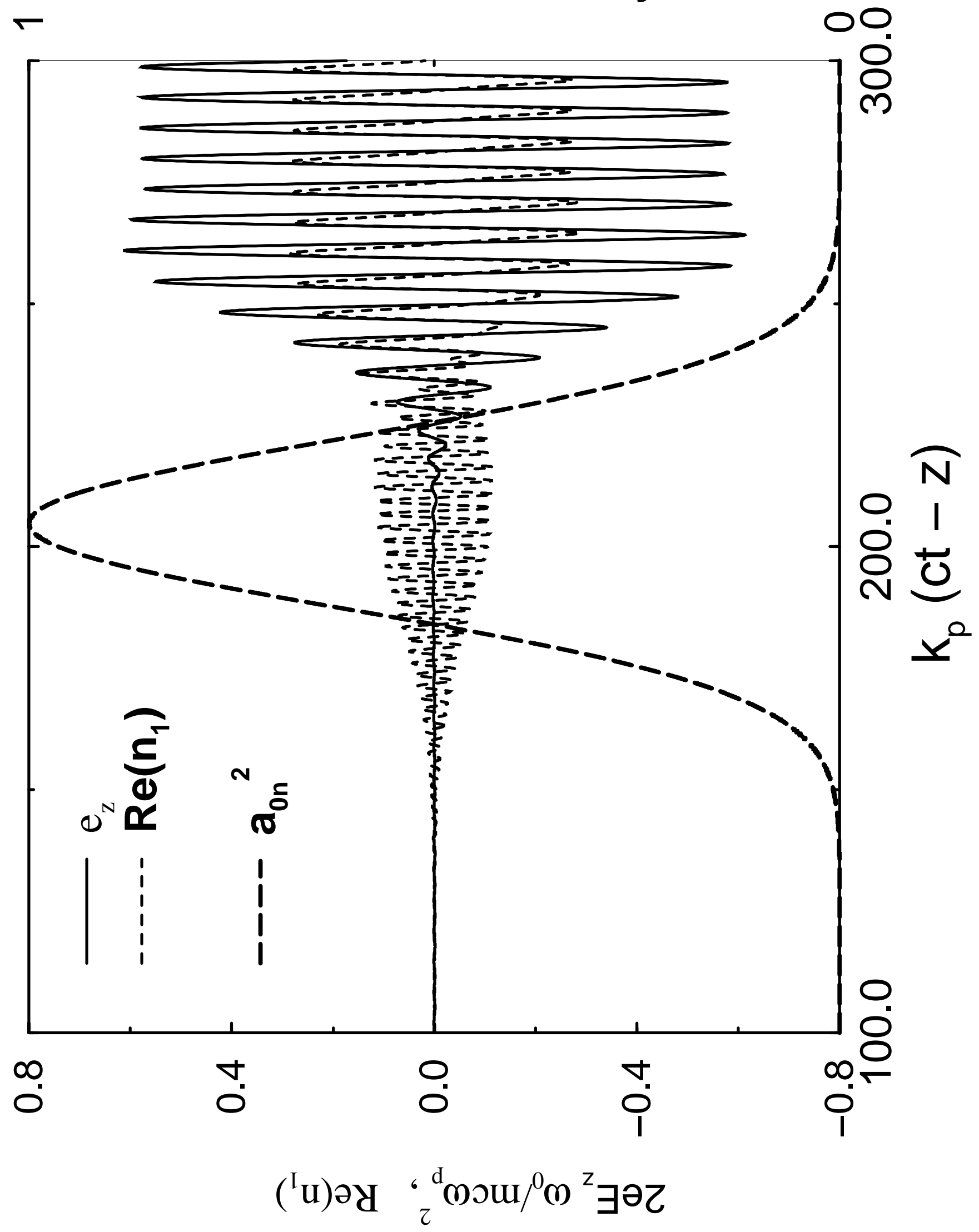




\section{External Distribution}

Plasma Research Laboratory, Australian National University, Australia

Professor I.R. J ones, Flinders University, Australia

Professor J oão Canalle, Instituto de Fisica DEQ/IF - UERJ , Brazil

Mr. Gerson O. Ludwig, Instituto Nacional de Pesquisas, Brazil

Dr. P.H. Sakanaka, Instituto Fisica, Brazil

The Librarian, Culham Laboratory, England

Library, R61, Rutherford Appleton Laboratory, England

Mrs. S.A. Hutchinson, JET Library, England

Professor M.N. Bussac, Ecole Polytechnique, France

Librarian, Max-Planck-Institut für Plasmaphysik, Germany

J olan Moldvai, Reports Library, MTA KFKI-ATKI, Hungary

Dr. P. Kaw, Institute for Plasma Research, India

Ms. P.J . Pathak, Librarian, Insitute for Plasma Research, India

Ms. Clelia De Palo, Associazione EURATOM-ENEA, I taly

Dr. G. Grosso, Instituto di Fisica del Plasma, Italy

Librarian, Naka Fusion Research Establishment, J AERI, J apan

Library, Plasma Physics Laboratory, Kyoto University, J apan

Research Information Center, National Institute for Fusion Science, J apan

Dr. O. Mitarai, Kyushu Tokai University, J apan

Library, Academia Sinica, Institute of Plasma Physics, People's Republic of China

Shih-Tung Tsai, Institute of Physics, Chinese Academy of Sciences, People's Republic of China

Dr. S. Mirnov, Triniti, Troitsk, Russian Federation, Russia

Dr. V.S. Strelkov, Kurchatov Institute, Russian Federation, Russia

Professor Peter Lukac, Katedra Fyziky Plazmy MFF UK, Mlynska dolina F-2, Komenskeho Univerzita, SK-842 15 Bratislava, Slovakia

Dr. G.S. Lee, Korea Basic Science Institute, South Korea

Mr. Dennis Bruggink, Fusion Library, University of Wisconsin, USA

Institute for Plasma Research, University of Maryland, USA

Librarian, Fusion Energy Division, Oak Ridge National Laboratory, USA

Librarian, Institute of Fusion Studies, University of Texas, USA

Librarian, Magnetic Fusion Program, Lawrence Livermore National Laboratory, USA

Library, General Atomics, USA

Plasma Physics Group, Fusion Energy Research Program, University of California at San Diego, USA

Plasma Physics Library, Columbia University, USA

Alkesh Punjabi, Center for Fusion Research and Training, Hampton University, USA

Dr. W.M. Stacey, Fusion Research Center, Georgia Institute of Technology, USA

Dr. J ohn Willis, U.S. Department of Energy, Office of Fusion Energy Sciences, USA

Mr. Paul H. Wright, Indianapolis, Indiana, USA 
The Princeton Plasma Physics Laboratory is operated by Princeton University under contract with the U.S. Department of Energy.

\author{
Information Services \\ Princeton Plasma Physics Laboratory \\ P.O. Box 451 \\ Princeton, NJ 08543
}

Phone: 609-243-2750

Fax: 609-243-2751

e-mail: pppl_info@pppl.gov

Internet Address: http://www.pppl.gov 